\title{
Family Health History and Behavioral Change among Undergraduate Students: A Mixed Methods Study
}

\author{
Ronica N. Rooks ${ }^{*}$, Cassandra D. Ford ${ }^{2}$ \\ ${ }^{1}$ Department of Health and Behavioral Sciences, University of Colorado Denver, Denver, USA \\ ${ }^{2}$ The Capstone College of Nursing, The University of Alabama, Tuscaloosa, USA \\ Email: *ronica.rooks@ucdenver.edu
}

Received 20 January 2016; accepted 23 February 2016; published 26 February 2016

Copyright (C) 2016 by authors and Scientific Research Publishing Inc.

This work is licensed under the Creative Commons Attribution International License (CC BY). http://creativecommons.org/licenses/by/4.0/

(c) (i) Open Access

\section{Abstract}

Background: We examined family health history (FHH) as a public health intervention tool in undergraduate students. We hypothesized that the FHH assignment would positively relate to students' FHH knowledge and health and healthcare-seeking behavioral change. Methods: Health professional students' $(n=103)$ pre/post-test surveys and research papers were collected in 2011-2012, from a mid-western and southern university in the United States of America, using mixed methods research. Results: The majority of students were aged 18 - 30, women, White, had healthcare access and health insurance, and awareness of the term FHH. Significant logistic regression relationships existed between: 1) helping students understand important strengths and weaknesses in their health and quality of life and outcomes of talking with family and doctors about FHH; and 2) improving students' understanding of what they needed to do to maintain their health and the outcome statement "FHH tells you about inherited genes." Key themes from the research papers included actions and FHH and proposed behavioral changes. Conclusions: Quantitative findings supported the relationship between students' assignment evaluation and knowledge change, while qualitative findings supported relationships between assignment evaluation and knowledge and behavioral change. This study highlights regional differences in students' FHH and the need to address family support barriers to behavioral change.

\section{Keywords}

Family Health History, Health Behaviors, Undergraduate Students, Health Professionals

\footnotetext{
${ }^{*}$ Corresponding author.
} 


\section{Introduction}

Family health history (FHH) can be used as a tool to assess health risk, improve data collection and disease prevention, and motivate behavioral change [1]. But, while $96.3 \%$ of those participating in the 2004 HealthStyles national survey thought family history was important for their own health, only $30 \%$ collected this information from relatives [2]. Using web-based FHH tools, such as the Surgeon General's My Family Health Portrait [3], FHH can be shared with family members and providers to target preventive health behaviors. But, using FHH as a public health tool has not been fully explored to improve health behaviors [1].

FHH is a risk factor for many chronic diseases, reflecting shared genetic susceptibility, environments, and common behaviors [4]. An accurate FHH knowledge may reduce disease risk by improving risk perceptions and positive health behaviors [5]. However, little research exists on whether collecting FHH is associated with measurable behavioral change [6].

At the conclusion of a two-month FHH intervention pilot study in adults, researchers found $84 \%$ of participants shared their FHH information with families, 5\% planned to share it with family, $79 \%$ planned to share their information with providers, and $80 \%$ planned to improve their diet and daily exercise [7]. Other FHH research targeting students and their families in a three-month intervention reported improved ability to openly communicate about family health issues [6]. Many students initiated conversations with healthcare providers and improved their physical activity and nutrition. However, little FHH intervention research targets undergraduate students as health professionals in training.

Understanding FHH is vital to students' career development and long-term health, since they need to understand and communicate its importance to future patients and/or researchers. Students are also at an age when chronic conditions often begin developing. Students' awareness of their FHH may enable them to concentrate on specific preventive behaviors.

As students examine their FHHs in the context of course and family discussions, they may improve their health behaviors. Studies have shown the importance of developing positive health behaviors at an early age, when these behaviors are more likely to become habitual [8]. These changes can also reduce long-term chronic condition risk [9] [10].

Social cognitive theory suggests that human behavior is an interactive process between personal, behavioral, and environmental influences, where people learn from their own and others' experiences through observational learning [11]. Behavioral changes are based on reinforcement, self-control, self-efficacy, and reciprocal determinism, with people acting as agents for and responders to change. In our research students respond to change based on our course curriculum and their learning from the FHH assignment. They are motivators for change as they discuss their FHH with family members. Moreover, students become health information seekers. Their learning about inherited diseases and treatments may influence if and how they use this information [12]. Health information use, for self or family, could improve health behaviors and patient-doctor interaction, avert preventable health conditions, and reduce health disparities [13]-[15].

The purpose of this mixed methods study was to identify changes in students' FHH knowledge and health and healthcare-seeking behaviors after classroom instruction and writing FHH research papers. The benefits of using FHH to promote training and behavioral change among health professional students include its easy accessibility; emphasis on promoting family interaction, which is cross-culturally applicable; and emphasis on engaged, problem-based student learning to benefit themselves and future patients. We hypothesized that by the post-test, assignment evaluation would be related to positive change in $\mathrm{H}_{1}$ ) FHH knowledge and $\mathrm{H}_{2}$ ) behaviors.

\section{Methodology}

\subsection{Study Design/Sample}

This study was conducted at a mid-western and southern university in the United States of America (USA). Undergraduate students aged 18+ were enrolled in the study from 2011-2012, through an elective public health course offered twice, over two years, and a required nursing course, where each course lasted one semester. Data consisted of students' pre-/post-test surveys and research papers. Pre-/post-tests focused on FHH knowledge, health and healthcare-seeking behaviors, and socio-demographic information. Students' research papers focused on a prominent disease, disorder, or disability discovered in their FHHs. The paper included: 1) a literature review on health disparities for their family disease outcome; 2) prevention and intervention programs for the disease; 3) a family relative interview; and 4) reflections on whether this assignment impacted their health beha- 
viors and any future actions. The sample included 136 students across both sites. However, difficulties matching anonymous pre-/post-tests for some students and course drops and/or absences on the post-test day reduced our sample to 103 students. Our qualitative analysis was based on a subsample of 65 students.

Faculty at each site verified students' consent form completion. Students could submit their consent forms to our research assistants after review in class or by the end of the semester. Students were assured their consent (or non-consent) would not impact their assignment or course grades. Public health students agreed to participate in this assignment by their elective course enrollment. However, the assignment was part of a required course for nursing students. At both sites, if students did not provide consents their surveys and papers were removed from the study. This study was approved by the Institutional Review Boards at each institution.

\subsection{Measures}

Outcomes. We included post-test FHH knowledge for $\mathrm{H}_{1}$ and health and healthcare-seeking behaviors for $\mathrm{H}_{2}$. For FHH knowledge participants were asked questions with Likert scale, true/false, and yes/no responses including: how likely they were to talk with family members about their FHH; how likely they were to talk with a doctor about their FHH; FHH tells you about inherited genes; FHH tells you which diseases you will certainly develop; FHH indicates an inherited tendency towards developing disease; FHH is based on a family's physical environment; and before today, had you heard of a FHH (only asked at pre-test) [16]? Due to our small sample size, we recoded the first two questions as strongly agree and somewhat agree (1) versus strongly disagree, somewhat disagree, and neutral (0).

For health and healthcare-seeking behaviors students were asked about their smoking, physical activities, delayed medical care, and length of time since last healthcare use [17]. Students were asked if they smoked approximately five packs of cigarettes in their entire life, and if yes, how often they now smoke cigarettes. They were asked if they stopped smoking for more than one day during the past 30 days because they were trying to quit. These questions were coded as current, previous, or never smoker. For leisure-time physical activities students were asked how often they did moderate or vigorous activities for $\geq 10$ minutes that caused sweating or increased breathing or heart rate. Responses were recoded as $\geq 3$ - 5 days a week (1) versus 1 - 2 days a week, a few times a month, and never (0). For delayed medical care students were asked if during the past 12 months they delayed getting care for any of the following reasons: couldn't get through on the telephone; couldn't get an appointment soon enough; waited too long to see the doctor; the clinic/doctor's office wasn't open when you could get there; and/or you didn't have transportation. Also, during the past 12 months was there delayed healthcare because of the expense, including: general healthcare or check-up, prescription medicines, mental healthcare or counseling, dental care, and/or eyeglasses. For both questions we coded students who mentioned delayed care for any reason (1) versus those who didn't delay care (0). For interacting with a healthcare professional about your health, students were asked how long was it since they last saw or talked to a doctor or other healthcare professional about their health? We recoded student responses for those with healthcare professional interactions $>1$ year ago or never (1) versus $\leq 1$ year ago $(0)$.

Exposures. For $\mathrm{H}_{1}$ we included pre-test $\mathrm{FHH}$ knowledge and post-test $\mathrm{FHH}$ assignment evaluation questions [18]. For $\mathrm{H}_{2}$ we included pre-test health or healthcare-seeking behaviors and post-test FHH assignment evaluation questions. Due to high correlations, we used one of four questions for students' assignment evaluation in each model including: if they agreed (1) or disagreed (0) that this assignment helped improve their understanding of what they needed to do to maintain their health; it changed their behavior towards positive health habits; it prepared them for discussing core health concepts with their families; and, it helped them understand important strengths and weaknesses in their health and quality of life.

Covariates. We included socio-demographic information on age, primary race/ethnicity, gender, family income, access to care vs. no usual healthcare, and having vs. not having health insurance.

\subsection{Statistical Analysis}

We used quantitative and qualitative methods to analyze students' pre-/post-tests and research papers. Using mixed methods is beneficial for data triangulation, providing generalizability, and contextual-depth to our analyses [19]. For quantitative analysis, we used SPSS v22. We ran frequencies for socio-demographic characteristics and cross-tabulations by site with chi-square tests (Table 1). We ran cross-tabulations for FHH knowledge and health behaviors by pre-vs. post-test responses by site (Table 2). We ran correlations to determine variable 
Table 1. Participants’ pre-test socio-demographic characteristics by site.

\begin{tabular}{|c|c|c|c|c|}
\hline & Total & Site 1 & Site 2 & p-value \\
\hline & $n=103$ & $n=33$ & $\mathrm{n}=70$ & \\
\hline \multicolumn{5}{|l|}{ Age } \\
\hline $18-30$ & $96.1 \%(99)$ & $93.9 \%(31)$ & $97.1 \%(68)$ & ns \\
\hline $31-50$ & $3.9 \%(4)$ & $6.1 \%(2)$ & $2.9 \%(2)$ & \\
\hline \multicolumn{5}{|l|}{ Sex } \\
\hline Male & $13.6 \%(14)$ & $21.2 \%(7)$ & $10.0 \%(7)$ & ns \\
\hline Female & $86.4 \%(89)$ & $78.8 \%(26)$ & $90.0 \%(63)$ & \\
\hline \multicolumn{5}{|l|}{ Race/Ethnicity } \\
\hline Asian/Pacific Islander & $4.9 \%(5)$ & $12.1 \%(4)$ & $1.4 \%(1)$ & $\mathrm{p} \leq 0.001$ \\
\hline Black/African-American & $6.8 \%(7)$ & $9.1 \%(3)$ & $5.7 \%(4)$ & \\
\hline White/Caucasian & $75.7 \%(78)$ & $42.4 \%(14)$ & $91.4 \%(64)$ & \\
\hline Latino/Hispanic & $5.8 \%(6)$ & $15.2 \%(5)$ & $1.4 \%(1)$ & \\
\hline American Indian/Native American & $1.9 \%(2)$ & $6.1 \%(2)$ & $0 \%(0)$ & \\
\hline Other & $4.9 \%(5)$ & $15.2 \%(5)$ & $0 \%(0)$ & \\
\hline \multicolumn{5}{|l|}{ Income } \\
\hline$<\$ 50,000$ & $28.2 \%(29)$ & $46.4 \%(13)$ & $25.4 \%(16)$ & $\mathrm{p}=0.047$ \\
\hline$\geq \$ 50,000$ & $60.2 \%(62)$ & $53.6 \%(15)$ & $74.6 \%(47)$ & \\
\hline Missing & $11.7 \%(12)$ & & & \\
\hline \multicolumn{5}{|l|}{ Access to care } \\
\hline Yes & $85.4 \%(88)$ & $68.8 \%(22)$ & $94.3 \%(66)$ & $\mathrm{p}=0.001$ \\
\hline No & $13.6 \%(14)$ & $31.2 \%(10)$ & $5.7 \%(4)$ & \\
\hline Missing/Don’t Know & $1.0 \%(1)$ & & & \\
\hline \multicolumn{5}{|l|}{ Health insurance } \\
\hline Yes & $88.3 \%(91)$ & $81.2 \%(26)$ & $92.9 \%(65)$ & $\mathrm{p}=0.079$ \\
\hline No & $10.7 \%(11)$ & $18.8 \%(6)$ & $7.1 \%(5)$ & \\
\hline Missing/Don’t Know & $1.0 \%(1)$ & & & \\
\hline
\end{tabular}

Source: Students in the Health, Culture, and Society and Foundations of Nursing courses, 2011-2012.

associations with our outcomes and create parsimonious models. We conducted logistic regression analyses to address $\mathrm{H}_{1}$, running separate models for each of the six post-test $\mathrm{FHH}$ knowledge questions as outcomes. For $\mathrm{H}_{1}$, we controlled for each pre-test FHH question, race/ethnicity, gender, income, and access to care at pre-test, and one FHH assignment evaluation question at post-test (Table 3). We conducted logistic regression analyses to address $\mathrm{H}_{2}$, running separate models for each of the five post-test health and healthcare-seeking behaviors as outcomes. For $\mathrm{H}_{2}$, we controlled for each pre-test behavior and the socio-demographic variables from $\mathrm{H}_{1}$ (not shown).

We used qualitative content analysis in Microsoft Excel [20]. We identified common themes in students' paper reflections and future actions, discussing their experiences with a disease condition; whether they or family members made or planned to make behavioral changes; motivation for changes; and barriers to change encountered during their assignment. To increase inter-rater reliability, responses were independently reviewed by two coders at each site. Our qualitative analysis proceeded as follows, we: 1) created a list of disease conditions from students' papers; 2) identified common themes to compare across this list; 3) recorded content or quotes in Excel; and 4) left the cell blank if a topic was not discussed.

\section{Results}

\subsection{Quantitative Analysis}

Table 1 describes participants' pre-test socio-demographic characteristics by site. Almost all students were aged 18 - 30, women, White, had access to care, and health insurance. Significant site differences existed by race/ 
Table 2. Participants' family health history knowledge and health and healthcare-seeking behaviors by pre-/post-test surveys and site.

\begin{tabular}{|c|c|c|c|c|c|c|c|c|c|c|}
\hline & & \multicolumn{3}{|c|}{ Total } & \multicolumn{3}{|c|}{ Site 1} & \multicolumn{3}{|c|}{ Site 2} \\
\hline & & Pre-test & Post-test & p-value & Pre-test & Post-test & p-value & Pre-test & Post-test & p-value \\
\hline \multicolumn{11}{|l|}{ Family Health History } \\
\hline \multirow[t]{2}{*}{$\begin{array}{l}\text { How likely are you to talk } \\
\text { with your family about FHH? }\end{array}$} & Likely & $\begin{array}{c}87.4 \% \\
(90)\end{array}$ & $\begin{array}{c}88.3 \% \\
(91)\end{array}$ & $\mathrm{p} \leq 0.001$ & $\begin{array}{c}84.8 \% \\
(28)\end{array}$ & $\begin{array}{l}84.8 \% \\
(28)\end{array}$ & $\mathrm{p}=0.002$ & $\begin{array}{c}88.6 \% \\
(62)\end{array}$ & $\begin{array}{c}90.0 \% \\
(63)\end{array}$ & $\mathrm{p} \leq 0.001$ \\
\hline & Unlikely & $\begin{array}{c}12.6 \% \\
(13)\end{array}$ & $\begin{array}{c}11.7 \% \\
(12)\end{array}$ & & $\begin{array}{l}15.2 \% \\
(5)\end{array}$ & $\begin{array}{l}15.2 \% \\
(5)\end{array}$ & & $\begin{array}{l}11.4 \% \\
(8)\end{array}$ & $\begin{array}{l}10.0 \% \\
(7)\end{array}$ & \\
\hline \multirow{2}{*}{$\begin{array}{l}\text { How likely are you to talk } \\
\text { with a doctor about FHH? }\end{array}$} & Likely & $\begin{array}{c}65.7 \% \\
(67)\end{array}$ & $\begin{array}{l}76.5 \% \\
(78)\end{array}$ & $\mathrm{p}=0.019$ & $\begin{array}{c}56.2 \% \\
(18)\end{array}$ & $\begin{array}{c}65.6 \% \\
(21)\end{array}$ & $\mathrm{p}=0.017$ & $\begin{array}{l}70.0 \% \\
(49)\end{array}$ & $\begin{array}{c}81.4 \% \\
(57)\end{array}$ & ns \\
\hline & Unlikely & $\begin{array}{c}34.3 \% \\
(35)\end{array}$ & $\begin{array}{c}23.5 \% \\
(24)\end{array}$ & & $\begin{array}{l}43.8 \% \\
(14)\end{array}$ & $\begin{array}{c}34.4 \% \\
(11)\end{array}$ & & $\begin{array}{c}30.0 \% \\
(21)\end{array}$ & $\begin{array}{c}18.6 \% \\
(13)\end{array}$ & \\
\hline \multirow{2}{*}{$\begin{array}{l}\text { FHH tells you about } \\
\text { inherited genes. }\end{array}$} & TRUE & $\begin{array}{c}92.2 \% \\
(94)\end{array}$ & $\begin{array}{c}84.3 \% \\
(86)\end{array}$ & $\mathrm{p} \leq 0.001$ & $\begin{array}{l}84.4 \% \\
(27)\end{array}$ & $\begin{array}{l}81.2 \% \\
(26)\end{array}$ & $\mathrm{p}=0.010$ & $\begin{array}{c}95.7 \% \\
(67)\end{array}$ & $\begin{array}{c}85.7 \% \\
(60)\end{array}$ & $\mathrm{p}=0.008$ \\
\hline & FALSE & $\begin{array}{c}7.8 \% \\
(8)\end{array}$ & $\begin{array}{c}15.7 \% \\
(16)\end{array}$ & & $\begin{array}{l}15.6 \% \\
(5)\end{array}$ & $\begin{array}{c}18.8 \% \\
(6)\end{array}$ & & $4.3 \%(3)$ & $\begin{array}{c}14.3 \% \\
(10)\end{array}$ & \\
\hline \multirow{2}{*}{$\begin{array}{l}\text { FHH tells which diseases } \\
\text { you will certainly develop. }\end{array}$} & TRUE & $\begin{array}{c}14.6 \% \\
(15)\end{array}$ & $\begin{array}{c}16.5 \% \\
(17)\end{array}$ & $\mathrm{p} \leq 0.001$ & $\begin{array}{l}18.2 \% \\
(6)\end{array}$ & $\begin{array}{l}15.2 \% \\
(5)\end{array}$ & $\mathrm{p}=0.008$ & $\begin{array}{l}12.9 \% \\
(9)\end{array}$ & $\begin{array}{c}17.1 \% \\
(12)\end{array}$ & $\mathrm{p} \leq 0.001$ \\
\hline & FALSE & $\begin{array}{c}85.4 \% \\
(88)\end{array}$ & $\begin{array}{c}83.5 \% \\
(86)\end{array}$ & & $\begin{array}{c}81.8 \% \\
(27)\end{array}$ & $\begin{array}{l}84.8 \% \\
(28)\end{array}$ & & $\begin{array}{c}87.1 \% \\
(61)\end{array}$ & $\begin{array}{c}82.9 \% \\
(58)\end{array}$ & \\
\hline \multirow{2}{*}{$\begin{array}{c}\text { FHH indicates an inherited } \\
\text { tendency towards } \\
\text { developing disease. }\end{array}$} & TRUE & $\begin{array}{c}98.0 \% \\
(100)\end{array}$ & $\begin{array}{c}99.0 \% \\
(101)\end{array}$ & ns & $\begin{array}{c}93.9 \% \\
(31)\end{array}$ & $\begin{array}{l}100.0 \% \\
(33)\end{array}$ & ns & $\begin{array}{c}100.0 \% \\
(69)\end{array}$ & $\begin{array}{c}98.6 \% \\
(68)\end{array}$ & ns \\
\hline & FALSE & $\begin{array}{c}2.0 \% \\
(2)\end{array}$ & $1.0 \%(1)$ & & $6.1 \%(2)$ & $\begin{array}{l}0.00 \% \\
(0)\end{array}$ & & $0.0 \%(0)$ & $\begin{array}{c}1.4 \% \\
(1)\end{array}$ & \\
\hline \multirow[t]{2}{*}{$\begin{array}{l}\text { FHH is based on a family's } \\
\text { physical environment. }\end{array}$} & TRUE & $\begin{array}{c}47.5 \% \\
(48)\end{array}$ & $\begin{array}{c}56.4 \% \\
(57)\end{array}$ & $\mathrm{p} \leq 0.001$ & $\begin{array}{c}51.5 \% \\
(17)\end{array}$ & $\begin{array}{c}57.6 \% \\
(19)\end{array}$ & $\mathrm{p}=0.024$ & $\begin{array}{c}45.6 \% \\
(31)\end{array}$ & $\begin{array}{c}55.9 \% \\
(38)\end{array}$ & $\mathrm{p}=0.005$ \\
\hline & FALSE & $\begin{array}{c}52.5 \% \\
(53)\end{array}$ & $\begin{array}{c}43.6 \% \\
(44)\end{array}$ & & $\begin{array}{c}48.5 \% \\
(16)\end{array}$ & $\begin{array}{l}42.4 \% \\
(14)\end{array}$ & & $\begin{array}{c}54.4 \% \\
(37)\end{array}$ & $\begin{array}{c}44.1 \% \\
(30)\end{array}$ & \\
\hline \multirow[t]{3}{*}{$\begin{array}{l}\text { Before today, had you heard } \\
\text { of FHH? }\end{array}$} & Yes & $\begin{array}{c}95.4 \% \\
(104)\end{array}$ & & & $\begin{array}{c}87.5 \% \\
(35)\end{array}$ & & & $\begin{array}{l}100.0 \% \\
(69)\end{array}$ & & \\
\hline & No & $\begin{array}{c}3.7 \% \\
(4)\end{array}$ & & & $\begin{array}{c}10.0 \% \\
(4)\end{array}$ & & & $\begin{array}{l}0.00 \% \\
(0)\end{array}$ & & \\
\hline & $\begin{array}{l}\text { Don't } \\
\text { Know }\end{array}$ & $\begin{array}{c}0.9 \% \\
(1)\end{array}$ & & & $2.5 \%(1)$ & & & $\begin{array}{l}0.00 \% \\
(0)\end{array}$ & & \\
\hline \multicolumn{11}{|l|}{$\begin{array}{c}\text { Health and } \\
\text { Healthcare-Seeking } \\
\text { Behaviors }\end{array}$} \\
\hline \multirow[t]{3}{*}{ Smoking } & Current & $\begin{array}{c}12.6 \% \\
(13)\end{array}$ & $\begin{array}{c}13.6 \% \\
(14)\end{array}$ & $\mathrm{p} \leq 0.001$ & $\begin{array}{l}12.1 \% \\
(4)\end{array}$ & $\begin{array}{l}12.1 \% \\
(4)\end{array}$ & $\mathrm{p} \leq 0.001$ & $\begin{array}{l}12.9 \% \\
(9)\end{array}$ & $\begin{array}{c}14.3 \% \\
(10)\end{array}$ & $\mathrm{p} \leq 0.001$ \\
\hline & Past & $\begin{array}{c}6.8 \% \\
(7)\end{array}$ & $\begin{array}{c}6.8 \% \\
(7)\end{array}$ & & $6.1 \%(2)$ & $6.1 \%(2)$ & & $7.1 \%(5)$ & $\begin{array}{l}7.1 \% \\
(5)\end{array}$ & \\
\hline & $\begin{array}{l}\text { Never } \\
\text { Smoked }\end{array}$ & $\begin{array}{c}80.6 \% \\
(83)\end{array}$ & $\begin{array}{c}79.6 \% \\
(82)\end{array}$ & & $\begin{array}{c}81.8 \% \\
(27)\end{array}$ & $\begin{array}{l}81.8 \% \\
(27)\end{array}$ & & $\begin{array}{c}80.0 \% \\
(56)\end{array}$ & $\begin{array}{c}78.6 \% \\
(55)\end{array}$ & \\
\hline \multirow{2}{*}{$\begin{array}{c}\text { Adequate moderate/vigorous } \\
\text { leisure time physical activity } \\
\text { in a wk.? }\end{array}$} & Yes & $\begin{array}{c}49.0 \% \\
(50)\end{array}$ & $\begin{array}{c}41.2 \% \\
(42)\end{array}$ & $\mathrm{p} \leq 0.001$ & $\begin{array}{c}42.4 \% \\
(14)\end{array}$ & $\begin{array}{c}45.5 \% \\
(15)\end{array}$ & $\mathrm{p}=0.062$ & $\begin{array}{c}52.2 \% \\
(36)\end{array}$ & $\begin{array}{c}39.1 \% \\
(27)\end{array}$ & $\mathrm{p} \leq 0.001$ \\
\hline & No & $\begin{array}{c}51.0 \% \\
(52)\end{array}$ & $\begin{array}{c}58.8 \% \\
(60)\end{array}$ & & $\begin{array}{c}57.6 \% \\
(19)\end{array}$ & $\begin{array}{c}54.5 \% \\
(18)\end{array}$ & & $\begin{array}{c}47.8 \% \\
(33)\end{array}$ & $\begin{array}{c}60.9 \% \\
(42)\end{array}$ & \\
\hline \multirow{2}{*}{$\begin{array}{l}\text { Did you delay seeking } \\
\text { healthcare in the last } \\
12 \text { mos? }\end{array}$} & Yes & $\begin{array}{c}16.5 \% \\
(17)\end{array}$ & $\begin{array}{c}18.4 \% \\
(19)\end{array}$ & $\mathrm{p} \leq 0.001$ & $\begin{array}{c}51.5 \% \\
(17)\end{array}$ & $\begin{array}{c}57.6 \% \\
(19)\end{array}$ & ns & $0.0 \%(0)$ & $\begin{array}{c}0.0 \% \\
(0)\end{array}$ & ns \\
\hline & No & $\begin{array}{c}83.5 \% \\
(86)\end{array}$ & $\begin{array}{c}81.6 \% \\
(84)\end{array}$ & & $\begin{array}{c}48.5 \% \\
(16)\end{array}$ & $\begin{array}{c}42.4 \% \\
(14)\end{array}$ & & $\begin{array}{c}100.0 \% \\
(70)\end{array}$ & $\begin{array}{c}100.0 \% \\
(70)\end{array}$ & \\
\hline \multirow{2}{*}{$\begin{array}{l}\text { Was there any time you } \\
\text { needed care but couldn’t } \\
\text { afford it in the last } 12 \text { mos.? }\end{array}$} & Yes & $\begin{array}{c}15.5 \% \\
(16)\end{array}$ & $\begin{array}{c}12.6 \% \\
(13)\end{array}$ & $\mathrm{p} \leq 0.001$ & $\begin{array}{c}48.5 \% \\
(16)\end{array}$ & $\begin{array}{c}39.4 \% \\
(13)\end{array}$ & $\mathrm{p}=0.055$ & $0.0 \%(0)$ & $0.0 \%(0)$ & ns \\
\hline & No & $\begin{array}{c}84.5 \% \\
(87)\end{array}$ & $\begin{array}{c}87.4 \% \\
(90)\end{array}$ & & $\begin{array}{c}51.5 \% \\
(17)\end{array}$ & $\begin{array}{c}60.6 \% \\
(20)\end{array}$ & & $\begin{array}{l}100.0 \% \\
(70)\end{array}$ & $\begin{array}{l}100.0 \% \\
(70)\end{array}$ & \\
\hline \multirow{2}{*}{$\begin{array}{l}\text { Longer than a year since last } \\
\text { interacted with a health } \\
\text { professional }\end{array}$} & Yes & $\begin{array}{c}11.8 \% \\
(12)\end{array}$ & $\begin{array}{l}8.8 \% \\
(9)\end{array}$ & $\mathrm{p} \leq 0.001$ & $\begin{array}{l}28.1 \% \\
\quad(9)\end{array}$ & $\begin{array}{l}21.9 \% \\
(7)\end{array}$ & $\mathrm{p}=0.004$ & $4.3 \%(3)$ & $2.9 \%(2)$ & ns \\
\hline & No & $\begin{array}{c}88.2 \% \\
(90)\end{array}$ & $\begin{array}{c}91.2 \% \\
(93)\end{array}$ & & $\begin{array}{c}71.9 \% \\
(23)\end{array}$ & $\begin{array}{c}78.1 \% \\
(25)\end{array}$ & & $\begin{array}{c}95.7 \% \\
(67)\end{array}$ & $\begin{array}{c}97.1 \% \\
(68)\end{array}$ & \\
\hline
\end{tabular}

Source: Students in the Health, Culture, and Society and Foundations of Nursing courses, 2011-2012. 
Table 3. Students' family health history assignment evaluation and knowledge change using logistic regression (ORs and $95 \%$ CIs).

\begin{tabular}{|c|c|c|c|c|}
\hline \multirow[b]{2}{*}{ Measures } & \multicolumn{3}{|c|}{ Models } & \\
\hline & 1 & 2 & 3 & 4 \\
\hline
\end{tabular}

FHH Knowledge at Pre-test

\begin{tabular}{|c|c|c|c|c|}
\hline $\begin{array}{l}\text { Likely to vs. not talk with } \\
\text { family about your FHH }\end{array}$ & $24.46^{* * *}(3.66,163.56)$ & & & \\
\hline $\begin{array}{l}\text { Likely to vs. not talk with } \\
\text { doctor about your FHH }\end{array}$ & & $4.23^{*}(1.34,13.35)$ & & \\
\hline $\begin{array}{c}\text { FHH tells you about } \\
\text { inherited genes vs. False/No }\end{array}$ & & & $26.55^{* *}(3.18,221.48)$ & \\
\hline $\begin{array}{c}\text { FHH is based on a family’s } \\
\text { physical environment vs. } \\
\text { False/No }\end{array}$ & & & & $5.21^{* *}(1.87,14.53)$ \\
\hline Other race vs. White & $0.61(0.10,3.92)$ & $0.46(0.12,1.79)$ & $0.23+(0.05,1.13)$ & $2.48(0.65,9.53)$ \\
\hline Men vs. Women & $0.27(0.04,1.92)$ & $5.29(0.53,52.82)$ & $1.21(0.15,10.13)$ & $0.20^{*}(0.05,0.90)$ \\
\hline $\begin{array}{c}\text { Family Income } \geq \$ 50,000 \text { vs. } \\
\text { less at Pre-test }\end{array}$ & $1.06(0.17,6.73)$ & $0.63(0.18,2.17)$ & $0.35(0.06,2.02)$ & $0.92(0.33,2.60)$ \\
\hline $\begin{array}{c}\text { Have Access to Care vs. } \\
\text { not at Pre-test }\end{array}$ & $4.61(0.41,52.16)$ & $2.04(0.40,10.40)$ & $0.11(0.01,1.95)$ & $1.62(0.34,7.71)$ \\
\hline \multicolumn{5}{|l|}{$\begin{array}{l}\text { Students' Evaluation of the } \\
\text { FHH Assignment at Post-test }\end{array}$} \\
\hline $\begin{array}{l}\text { Assignment helped you } \\
\text { anderstand important strengths } \\
\text { and weaknesses in your health } \\
\text { and quality of life vs. No }\end{array}$ & $18.83^{* *}(2.00,177.57)$ & $4.66^{+}(0.90,24.17)$ & & \\
\hline $\begin{array}{l}\text { Assignment improved my } \\
\text { anderstanding of what I need to } \\
\text { lo to maintain my health vs. No }\end{array}$ & & & $15.65^{+}(0.97,253.77)$ & \\
\hline $\begin{array}{l}\text { Assignment prepared me for } \\
\text { discussing core concepts of } \\
\text { health with my family vs. No }\end{array}$ & & & & $2.78(0.82,9.40)$ \\
\hline -2 Log Likelihood & 43.25 & 80.62 & 59.07 & 102.86 \\
\hline Nagelkerke R Square & 0.44 & 0.26 & 0.32 & 0.26 \\
\hline
\end{tabular}

Source: Students in the Health, Culture, and Society and Foundations of Nursing courses, 2011-2012, ${ }^{+} \mathrm{p} \leq 0.10 ;{ }^{*} \mathrm{p} \leq 0.05 ;{ }^{* *} \mathrm{p} \leq 0.01 ;{ }^{* * *} \mathrm{p} \leq 0.001$, Note. Models using the questions "FHH tells you which diseases you will certainly develop" and "FHH indicates an inherited tendency towards developing disease,” were not shown due to unstable odds ratios.

ethnicity $(p \leq 0.001)$, family income $(p=0.047)$, and access to care $(p=0.001)$. A marginally significant difference existed by site for students who had health insurance $(\mathrm{p}=0.079)$. There were no significant differences in students' ages and gender by site.

Table 2 describes participants' FHH knowledge and health and healthcare-seeking behaviors by pre-/posttests and site. Most students had heard of FHH prior to the pre-test. Participants were significantly more likely to speak with their family members $(\mathrm{p} \leq 0.001)$ and doctors $(\mathrm{p}=0.019)$ about their personal health issues from preto post-test. Participants significantly decreased their agreement over time on the question relating FHH to inherited genes ( $\mathrm{p} \leq 0.001)$. But, participants significantly increased their agreement on the questions relating FHH to diseases you will certainly develop $(p \leq 0.001)$ and a family's physical environment $(p \leq 0.001)$. Site 1 significantly decreased their agreement with the belief that FHH tells you which diseases you will certainly develop $(p=0.008)$. No significant change occurred over time for the belief that FHH indicates an inherited tendency towards disease.

Significant differences existed for all health and healthcare-seeking behaviors from pre-to post-test. The percentage of participants who had adequate exercise in a week ( $\mathrm{p} \leq 0.001)$, needed healthcare but could not afford it ( $\mathrm{p} \leq 0.001)$, and interacted with a healthcare professional $\geq 1$ year ago or never $(\mathrm{p} \leq 0.001)$ significantly decreased over time. However, participants who were current smokers $(\mathrm{p} \leq 0.001)$ and delayed care for any reason 
( $\leq 0.001)$ increased over time. Students in site 1 were slightly more likely to increase their exercise over time $(p=0.062)$. At site 2 , there were no significant differences in delayed care for any reason or needing healthcare but could not afford it over time; no students delayed care at the pre-or post-test.

Table 3 describes the relationship between students' assignment evaluation and knowledge change using logistic regression. The models focused on pre-test talking with family and doctors about FHH, FHH tells you about inherited genes, and FHH is based on a family's physical environment had significant relationships with the same post-test questions. Belief in the assignment helping students understand important strengths and weaknesses in their health and quality of life was significantly related to the likelihood of students talking with their families about FHH [OR:18.83; 95\% CI $(2.00,177.57)$; $\mathrm{p}=0.010]$. The same assignment evaluation question was marginally related to the likelihood of students talking with their doctors about FHH [OR: 4.66; 95\% CI (0.90, 24.17); $\mathrm{p}=0.067]$. Belief in the assignment improving students' understanding of what they need to do to maintain their health [OR: 15.65 ; 95\% CI $(0.97,253.77) ; \mathrm{p}=0.053$ ] and being of another race vs. White [OR: 0.23 ; $95 \%$ CI $(0.05,1.13)$; $\mathrm{p}=0.071]$ were marginally related to the statement that FHH tells students about inherited genes. Belief in the assignment preparing students for discussing core health concepts with their families was not significantly related to the statement that FHH is based on a family's physical environment. However, gender was significantly related [OR: 0.20 ; $95 \%$ CI $(0.05,0.90) ; \mathrm{p}=0.036$ ]. The models using outcomes of "FHH tells you which disease you will certainly develop" and "FHH indicates an inherited tendency towards developing disease" had unstable results; thus, we did not report these findings.

The relationships between students' assignment evaluation belief that it changed their behavior towards positive health habits and the post-test health and healthcare-seeking behaviors were examined using logistic regression. However, assignment evaluation was not significantly related to any of these outcomes (results not shown).

\subsection{Qualitative Analysis}

Analysis of students' papers from both sites provided insight into two major areas of discussion: actions and FHH and proposed behavioral changes. The conditions cited most often by students were obesity, Alzheimer's disease, cancer, type II diabetes, alcoholism, mental health disorders, and cardiovascular disease (including hypertension and myocardial infarction). Regional differences existed in some conditions reported, with more cardiovascular disease and related risk factors mentioned at the southern university.

\subsubsection{Actions}

Disease awareness and education reflected an increased need for prevention and treatment knowledge about chronic conditions. For some conditions, students reported a need for increased disease awareness. With Alzheimer's disease as a potentially early onset condition, one student commented on the need for public education/awareness, more funding and research for this disease as the "the most important action" to be taken. Students believed that education about a condition, including prevention and treatment options, should be more widely available to patients and the public. One student believed, "...education and awareness of public health issues is the first step to good prevention." Another student stated, "My eyes have been open [ed] to the fact of how important taking care of your body [is] not only when you are older, but also when you are young for that is the foundation for which you have to build upon as you grow up."

Behavioral changes provided insight into whether students changed their behavior over the course of the assignment and challenges they encountered. Almost all students reported some behavioral change for the conditions examined. But, most of the reported actions were proposed behavioral changes (considered under the second theme). Behavioral changes included feeling more confident in oneself, regular exercise, improved diet, smoking cessation, stress reduction, limiting alcohol consumption, and taking appropriate medication. One student learned she could offset Alzheimer's development by not smoking, regularly exercising, reducing stress, and proper nutrition. Another student's diet change was the daily use of St. John's Wort supplements, which some associate with depression treatment. Student challenges to behavioral change included: genetics, busy schedules, public policy, unaffordable medical care, and motivation. However, one student made an encouraging statement: "While my family has a predisposition for diabetes, we hold the power in our hands. Realizing how many people in my family have been diagnosed with diabetes is a huge factor in motivating my health oriented life. A few life-choice sacrifices now (like putting down the cheeseburger and taking the stairs) are worth not living with co-morbidities later.” 
Stigma reduction reflected students' beliefs in social stigmas attached to family members' conditions, particularly for mental illness. Students mentioned reducing stigma in the context of needing more disease awareness and education for the public and health professionals. One respondent explained, “...the best action to be taken would be to lift the stigma and have a more open dialogue about depression so that members of my family can better understand each other and create a stronger support system.” Another student stated, “....many people with TTM [Trichotilliomania] are embarrassed, shameful, and isolate themselves, so it's important that doctors are informed, educated, and reliable....”

Students mentioned stigma inhibiting recovery and treatment. One student explained, "People who are obese frequently feel the pressure to lose weight from the people and physicians around them, yet don't feel like they have a safe environment to facilitate these changes." Stigma was discussed in relation to alcoholism: "Public awareness should increase concerning the genetic predisposition to becoming alcohol dependent...society needs to acknowledge that alcohol dependence is a disease, and many individuals are not able to abruptly stop drinking...educating members of society will reduce the stigma associated with alcohol dependence and encourage individuals to seek help..."

Family/social support included having strong support or lacking it. The ability to discuss conditions with family, community members, or medical personnel was viewed as having strong support. Some supportive community programs were: Alcoholics Anonymous, LIVESTRONG, and I < 3 BOOBIES. For responses indicating poor support, the majority involved the inability to discuss conditions openly within families. One student recognized this as a family issue: “...the number one step...to change anything is to admit there is a problem. My family has a hard time admitting what they're doing wrong, and admitting that they are slowly killing themselves.” Another student described his/her home environment: "The way that my family deals with things is that we don't talk about it. Since I was little we have always been the type of family that doesn't approach problems when there is one.” A third student stated, "After completing this assignment I have a greater motivation to put my plan into action. My only concern is that my family won't be on board and willing to change with me. Holidays and special gatherings could possibly set my efforts back. However, I am going to continue to try to influence my family to make this important change with me.”

\subsubsection{FHH and Proposed Behavioral Changes}

Reflections on FHH discussed how family genes currently affect students, mostly for chronic, non-communicable diseases. One student noted, "I have learned how important knowing your FHH is through writing this paper. When I have my own family, I will be sure to educate my children about being heart healthy and ways to prevent heart disease.”

Cultural competency reflected the need for it within the medical field. A small number of students expressed this need, particularly for mental illness. Reflecting on schizophrenia one student stated, "For an ethnic or racial minority, it is important to go to a hospital where the minority can properly communicate with the doctors. Racial and ethnic minorities should not hesitate in asking questions if they are not satisfied with the quality of care and they should be determined in finding a doctor that will give them exactly what they are looking for.” This student believed patients have some responsibility in receiving high quality healthcare by actively asking questions.

Proposed behavioral change reflected changes that have not yet occurred at the individual and community levels. Students mentioned proposed behavioral changes in diet and exercise, such as using Eastern, homeopathic remedies to combat depression, receiving frequent health screenings, and finding outlets to express feelings and ideas. Many proposed community-level behavioral changes included interventions to increase disease awareness and reduce stigma, such as having block parties and other social events to support cancer patients and their family/friends. "This way cancer patients have the opportunity to be exposed to others sharing their experience...the larger idea here is that cancer patients and those without cancer are mingling together in a stress-free environment...This....would ideally reduce stigma associated with cancer patients and promote better self-image for the patients...” Another community-level change mentioned for depression was to change media portrayals of depression and anti-depressants.

After learning about a FHH of heart disease and seeing a visual representation of its effects, one student stated he/she would screen and inform the doctor about his/her FHH. Another student stated the assignment positively impacted the health behaviors of his/her family. The student stated, "Now that I know more about a hereditary disease that many people in my family have experienced I am going to start being more aware of my negative 
health behaviors, and make more of an effort to include more positive behaviors in my life.” One student hoped future generations would look at his/her FHH and learn from past family mistakes and correct choices.

\section{Discussion}

This study provided insight about undergraduate health professional students and relationships between assignment evaluation and their FHH knowledge and behavioral changes. Significant logistic regression relationships existed between students' understanding: 1) strengths and weaknesses in their health and talking with family and doctors about FHH and 2) what they needed to do to maintain their health and the statement "FHH tells you about inherited genes." Qualitative themes from students' papers included actions and FHH and proposed behavioral changes. Under actions, students discussed disease awareness and education, behavioral changes, stigma, and family/social support. For FHH, students discussed their reflections, cultural competency, and proposed behavioral changes. We found partial support in our quantitative findings for the relationship between assignment evaluation and knowledge change, while our qualitative findings supported the relationships between assignment evaluation and knowledge and behavioral change.

The assignment reminded students of their power to make changes to address family diseases. Students discussed this assignment as an opportunity for communication with family members and taking a more proactive role in their health [6]. Students' reflections related to social cognitive theory, where students learned through their research experiences by observing others' actions during family interviews and subsequent health interactions.

Conducting this study in two areas of the country, with different types of health professional students, lead to some site differences. The southern, nursing sample was larger than the mid-western, public health sample, possibly because the nursing course was required. However, more nursing than public health students were excluded from our final sample because their pre-/post-tests could not be matched, despite having high student participation with consents. Students also discussed different conditions in their papers, particularly the high rate of cardiovascular disease in the southern USA [21]. However, students at both sites noted concern about family members accepting their lifestyle changes or if a lack of support would be a barrier to behavioral change. This finding is reflective of other studies discussing a healthy lifestyle, behavioral change, and social support [22] [23].

We encountered some unique student situations making this assignment and data collection challenging. For example, one student did not have living biological family. After careful discussion, the student chose to research the disease that most affected his/her family and resulted in their deaths. We had an adopted student and one who was estranged from his/her family. We asked them to focus on "nurture" issues of adoptive family and broader community socialization influencing their health behaviors and beliefs.

Over time, we refined our data collection techniques to enhance our pre-/post-test matching and added more assignment discussions with students. We were frustrated by our efforts to maintain student anonymity in the pre-/post-tests while attempting to match them. One solution is using electronic surveys; however, this strategy could decrease our sample size. Finally, we learned to better describe our research project to students, improving their comfort with us anonymously using their papers for research, using third-party consenters, and clarifying what signed consent means.

Future research may include learning more about family and provider prompts to FHH-related behavioral change. While we did not collect information on family members' behavioral changes resulting from students' interviews, these interactions could influence students' behavioral changes. Related to social cognitive theory, we could consider if the number of people with diseases in a family; examining the lives of those living with these diseases; the number of deaths from these diseases; or working as a family unit to make collective behavioral changes influence students' behavioral changes. We wondered if the number of FHH prompts from healthcare providers or their suggestions to use FHH tools fosters proactive thinking about family conditions and influences students' behavioral changes.

This study had some limitations. First, data collection was completed during one semester for each course. It is difficult to identify behavioral change in one semester; however, research shows that habits are generally formed within 30 days [24]. For a longer evaluation of students' FHH and proposed behavioral changes, researchers could get consent from students to conduct email follow-ups six months after the course ends. Questions should focus on understanding if and how students maintained their behavioral change(s) and if these changes aligned with their expectations at the end of the course. Second, the generalizability of our findings may 
be limited to undergraduate, health professional students. Third, post-test health behaviors could be influenced by intense coursework and increased stress levels at the end of the semester. Fourth, FHH information was self-reported; however, similar data are collected by healthcare providers. Lastly, some students encountered difficulties discussing their FHH with family members. We made suggestions to students, including sharing information about the course assignment to begin the conversation.

\section{Conclusion}

This study provided an opportunity to explore FHH as a tool to change students' knowledge and health behaviors, as well as enhance professional development. Electronic FHH tools are cost effective and accessible, while supporting comprehensive care and chronic condition prevention. Changing health behaviors via a course assignment in early adulthood, a life-course stage when students are still shaping their behaviors and chronic diseases begin developing, can result in a healthier lifestyle with long-term changes.

\section{Acknowledgements}

R. N. R. and C. D. F. thank our research assistants for data analyses help, including: Nathaniel Gage, Annika Jones, Al-Karim Gilani, and Michael Walker. We also thank our research assistants for manuscript formatting assistance, including: Helpees Guirguis and Britanie Pfeifer. This work was supported by internal funding (Advancing Curricula and Teaching and the Dean's Fund for Excellence awards) from one of our participating universities.

\section{Declaration of Interests}

The authors declare that they have no competing interests.

\section{References}

[1] Valdez, R., Yoon, P.W., Qureshi, N., Green, R.F. and Khoury, M.J. (2010) Family History in Public Health Practice: A Genomic Tool for Disease Prevention and Health Promotion. Annual Review of Public Health, 31, 69-87. http://dx.doi.org/10.1146/annurev.publhealth.012809.103621

[2] Gwinn, M., Hariri, S., Jorgensen, C., Khoury, M.J., Lyn, S., Scheuner, M.T. and Yoon, P.W. (2004) Awareness of Family Health History as a Risk Factor for Disease-United States, 2004. Morbidity and Mortality Weekly Report, 53, 1044-1047.

[3] My Family Health Portrait: A Tool from the Surgeon General. (2016) https://familyhistory.hhs.gov/fhh-web/home.action

[4] Yoon, P.W., Scheuner, M.T., Peterson-Oehlke, K.L., Gwinn, M., Faucett, A. and Khoury, M.J. (2002) Can Family History be Used as a Tool for Public Health and Preventive Medicine? Genetics in Medicine, 4, 304-310. http://dx.doi.org/10.1097/00125817-200207000-00009

[5] Murthy, V.S., Garza, M.A., Almario, D.A., Vogel, K.J., Grubs, R.E., Gettig, E.A., Wilson, J.W. and Thomas, S.B. (2011) Using a Family History Intervention to Improve Cancer Risk Perception in a black Community. Journal of Genetic Counseling, 20, 639-649. http://dx.doi.org/10.1007/s10897-011-9389-2

[6] Moore, P.J., Gratzer, W., Lieber, C., Edelson, V., O’Leary, J. and Terry, S.F. (2012) Iona College Community Centered Family Health History Project: Lessons Learned from Student Focus Groups. Journal of Genetic Counseling, 21, 127-135. http://dx.doi.org/10.1007/s10897-011-9392-7

[7] Petruccio, C., Mills Shaw, K.R., Boughman, J., Fernandez, C., Harlow, I., Kruesi, M., Kyler, P., Lloyd-Puryear, M.A., O’Leary, J., Skillman, A., et al. (2008) Healthy Choices through Family History: A Community Approach to Family History Awareness. Community Genetics, 11, 343-351.

[8] Aarts, H., Paulussen, T. and Schaalma, H. (1997) Physical Exercise Habit: On the Conceptualization and Formation of Habitual Health Behaviours. Health Education Research, 12, 363-734. http://dx.doi.org/10.1093/her/12.3.363

[9] Strong, K.A., Parks, S.L., Anderson, E., Winett, R. and Davy, B.M. (2008) Weight Gain Prevention: Identifying TheoryBased Targets for Health Behavior Change in Young Adults. Journal of the American Dietetic Association, 108, 17081715. http://dx.doi.org/10.1016/j.jada.2008.07.007

[10] Wantland, D.J., Portillo, C.J., Holzemer, W.L., Slaughter, R. and McGhee, E.M. (2004) The Effectiveness of WebBased vs. Non-Web-based Interventions: A Meta-Analysis of Behavioral Change Outcomes. Journal of Medical Internet Research, 6, e40. http://dx.doi.org/10.2196/jmir.6.4.e40 
[11] Glanz, P. and Rimer, B.K. (2005) Theory at a Glance: A Guide for Health Promotion Practice. 2nd Edition, U.S. Dept. of Health and Human Services, Public Health Service, National Institutes of Health, National Cancer Institute.

[12] Czaja, R., Manfredi, C. and Price, J. (2003) The Determinants and Consequences of Information Seeking among Cancer Patients. Journal of Health Communication, 8, 529-562. http://dx.doi.org/10.1080/716100418

[13] Eng, T.R., Maxfield, A., Patrick, K., Deering, M.J., Ratzan, S.C. and Gustafson, D.H. (1998) Access to Health Information and Support: A Public Highway or a Private Road? Journal of the American Medical Association, 280, 13711375. http://dx.doi.org/10.1001/jama.280.15.1371

[14] Hibbard, J.H., Greene, J., Becker, E.R., Roblin, D., Painter, M.W., Perez, D.J., Burbank-Schmitt, E. and Tusler, M. (2008) Racial/Ethnic Disparities and Consumer Activation in Health. Health Affairs (Millwood), 27, 1442-1453. http://dx.doi.org/10.1377/hlthaff.27.5.1442

[15] Lewis, N., Gray, S.W., Freres, D.R. and Hornik, R.C. (2009) Examining Cross-Source Engagement with CancerRelated Information and Its Impact on Doctor-Patient Relations. Health Communication, 24, 723-734. http://dx.doi.org/10.1080/10410230903264030

[16] Kaphingst, K.A., Lachance, C.R., Gepp, A., D’Anna, L.H. and Rios-Ellis, B. (2011) Educating Underserved Latino Communities about Family Health History using Lay Health Advisors. Public Health Genomics, 14, 211-221. http://dx.doi.org/10.1159/000272456

[17] National Center for Health Statistics, Centers for Disease Control and Prevention (2010) National Health Interview Survey, 2009 Sample Adult File.

[18] Rakel, D.P. and Hedgecock, J. (2008) Healing the Healer: A Tool to Encourage Student Reflection Towards Health. Medical Teacher, 30, 633-635. http://dx.doi.org/10.1080/01421590802206754

[19] Borkan, J.M. (2004) Mixed Methods Studies: A Foundation for Primary Care Research. Annals of Family Medicine, 2, 4-6. Http://Dx.Doi.Org/10.1370/Afm.111

[20] Swallow, V., Newton, J. and Van Lottum, C. (2003) How to Manage and Display Qualitative Data Using “Framework” and Microsoft Excel. Journal of Clinical Nursing, 12, 610-612. http://dx.doi.org/10.1046/j.1365-2702.2003.00728.x

[21] Go, A.S., Mozaffarian, D., Roger, V.L., Benjamin, E.J., Berry, J.D., Borden, W.B., Bravata, D.M., Dai, S., Ford, E.S., Fox, C.S., et al. (2013) Heart Disease and Stroke Statistics-2013 Update: A Report from the American Heart Association. Circulation, 127, e6-e245. http://dx.doi.org/10.1161/cir.0b013e31828124ad

[22] Ford, C.D., Kim, M.J. and Dancy, B.L. (2009) Perceptions of Hypertension and Contributing Personal and Environmental Factors among Rural Southern African American Women. Ethnicity \& Disease, 19, 407-413.

[23] James, D.C. (2004) Factors Influencing Food Choices, Dietary Intake, and Nutrition-Related Attitudes among African Americans: Application of a Culturally Sensitive Model. Ethnicity and Health, 9, 349-367. http://dx.doi.org/10.1080/1355785042000285375

[24] Gelmon, S.B., Holland, B.A., Driscoll, A., Spring, A. and Kerrigan, S. (2001) Assessing Service-Learning and Civic Engagement: Principles and Techniques. Brown University: Campus Compact, Providence, RI. 